\title{
Producción científica de los Institutos y Centro de Investigación de la Facultad de Medicina de la Universidad Nacional Mayor de San Marcos
}

Research Institutes/Center scientific production at the Faculty of Medicine of Universidad Nacional Mayor de San Marcos

\author{
José Pacheco-Romero, ${ }^{1, a}$, Mg. Doris Huerta ${ }^{2, b}$, Carlos Galarza, ${ }^{3, a}$, \\ José Ramos-Castillo
}

1 Profesor, Obstetricia y Ginecología; Instituto de Investigaciones Clínicas, Facultad de Medicina, Universidad Nacional Mayor de San Marcos. 2 Profesora, Bioquímica; Centro de Investigación en Bioquímica y Nutrición, Facultad de Medicina, Universidad Nacional Mayor de San Marcos. ${ }^{3}$ Profesor, Medicina; Instituto de Investigaciones Clínicas, Facultad de Medicina, Universidad Nacional Mayor de San Marcos. ${ }^{4}$ Médico Cirujano. Facultad de Medicina, UNMSM. Medico, Policlínico Fiori, Red Asistencial Sabogal, EsSalud. Lima, Perú.

\begin{abstract}
Resumen
Objetivos: Determinar la producción científica de los investigadores de la Facultad de Medicina, UNMSM, y la publicación de sus investigaciones, durante los años 2004-2009. Diseño: Estudio retrospectivo, observacional, de corte transversal. Institución: Facultad de Medicina, Universidad Nacional Mayor de San Marcos, Lima, Perú. Participantes: Investigadores de los seis Institutos de Investigación y un Centro de Investigación. Intervenciones: Evaluación de las fichas del investigador elaboradas por la Unidad de Investigación de la Facultad y llenadas por los investigadores de los Institutos de Investigación de Biología Andina, Cirugía Experimental, Ética en Salud, Investigaciones Clínicas, Medicina Tropical, Patología y el Centro de Investigación en Bioquímica y Nutrición, de la Facultad de Medicina. Para el análisis de datos de empleó el sistema Epi-Info versión 3.5.1 y el modelo estadístico Anova para el análisis de varianza. Principales medidas de resultados: Investigaciones realizadas y publicadas durante los años 2004-2009. Resultados: De los 116 docentes investigadores de los seis Institutos y un centro de investigación, solo llenaron la ficha del investigador 54, de cinco Institutos y el centro, quienes señalaron que habían realizado 659 trabajos de investigación durante los seis años, siendo el promedio dos investigaciones por investigador-año. Publicaron un total de 393 artículos, que representó un promedio de 7,2 por investigador durante los seis años de estudio y 1,2 por investigador-año; $343(87,3 \%)$ investigaciones fueron publicadas en revistas nacionales y $50(12,7 \%)$ en revistas internacionales; $76 \%$ de quienes respondieron aseguró que alguna de sus publicaciones había tenido impacto, principalmente en salud. Diecinueve por ciento de los investigadores no realizó publicación alguna en dicho lapso. No hubo diferencia significativa del género del investigador, grado académico, clase docente, profesión o membresía en alguno de los Institutos/Centro, aunque el promedio de investigaciones del Instituto de Investigaciones Clínicas fue mayor al resto. Conclusiones: Entre 2004 y 2009, los docentes-investigadores de la Facultad de Medicina realizaron 659 trabajos de investigación, con un promedio de dos investigaciones por investigador-año. Diecinueve por ciento de los investigadores no realizó publicación alguna en dicho lapso, y quienes publicaron lo hicieron siete veces más en revistas nacionales que en internacionales.

Palabras clave: Investigación biomédica, investigadores, docentes médicos, Facultad de Medicina/Universidad Nacional Mayor de San Marcos.

Abstract

Objectives: To determine research production at the Faculty of Medicine, UNMSM. Design: Retrospective, observational, transversal type study. Setting: Faculty of Medicine, Universidad Nacional Mayor de San Marcos, Lima, Peru. Participants: Seven research institutes and one investigation center investigators. Interventions: Study of investigator cards elaborated by the Faculty of Medicine Research Unit and filled by members of the Andean Biology, Experimental Surgery, Ethics in Health, Clinical Investigations, Tropical Medicine, and Pathology Research Institutes as well as by Biochemistry and Nutrition Investigation Centre members. Data analysis used EpiInfo system version 3.5.1 and Anova statistical model for analysis of variance. Main outcome measures: Scientific investigations and publications. Results: From the 116 members of the six Research Institutes/Centre only 54 from five Institutes and the Centre filled the investigator card and reported they had done 659 investigations during the six-year period and published an average of two investigations per investigator-year; $19 \%$ never published during the period studied. There was no significant difference of investigators gender, academic degree, teaching hours, profession or Institute/Centre, but members of one Institute seemed to investigate more. Conclusions: Docents-researchers filling the card reported 659 investigations between 2004-2009 averaging two investigations per researcher-year. One of the Institutes seemed to have done more research. Nineteen per cent of the investigators did not publish during those years and those publishing did it seven times more in national than international journals.
\end{abstract}

Key words: Biomedical research, research personnel, faculty/medical, Faculty of Medicine/Universidad Nacional Mayor de San Marcos.

An Fac med. 2012;73(2):147-51 


\section{INTRODUCCIÓN}

La investigación científica es la búsqueda intencionada de conocimientos o de soluciones a problemas de carácter científico $^{(1)}$, que tiene por fin ampliar el conocimiento científico, sin perseguir, en principio, ninguna aplicación práctica ${ }^{(2)}$. Es un conjunto de procesos sistemáticos, críticos y empíricos que se aplican al estudio de un fenómeno ${ }^{(3)}$. Una investigación se caracteriza por ser un proceso sistemático, organizado y objetivo ${ }^{(4)}$. La investigación en el Perú es considerada limitada, personalizada, direccionada, de calidad científica limitada y sin el apoyo estatal o institucional, y más bien parte de la iniciativa personal. Los recursos para la investigación en medicina son escasos, dirigida por instituciones internacionales, estatales y particulares nacionales y laboratorios científicos con interés en medicamentos o equipos y materiales en investigación.

El Vicerrectorado de Investigación de la Universidad Nacional Mayor de San Marcos fue creado en junio de 2005, mediante la RR N ${ }^{\circ}$ 03316-R-05, tras la decisión estratégica de la UNMSM de convertirse en la universidad líder en producción de conocimientos del país, respondiendo así a las expectativas y requerimientos de la sociedad peruana ${ }^{(5)}$. Así, el deseo de impulsar la investigación en la universidad ha significado el ordenamiento de la investigación, el monitoreo, la creación de puntajes del investigador de acuerdo a la producción y publicación científica, el estímulo para los investigadores de las diversas facultades y, particularmente en la Facultad de Medicina, la organización de talleres de capacitación y el perfeccionamiento de los concursos de investigación, con apoyo económico, pero también con el compromiso de informes técnicos y económicos de acuerdo a plazos establecidos.

En la Facultad de Medicina de la UNMSM, en el último quinquenio, se han organizado talleres dirigidos a capacitación en investigación, y en abril de 2009 se realizó con el apoyo académico de la Organización Panamericana de la Salud, un Taller de Análisis Estratégico sobre Investigación en la Facultad de Medicina de San Fernando. Las conclusiones del taller estratégico, entre otros, señalaban la necesidad de conocer la producción de cada investigador de los diversos Institutos de Investigación de la Facultad, de manera que los resultados permitieran tomar las medidas de perfeccionamiento en investigación.

No habiéndose determinado anteriormente el número de investigaciones realizadas y publicadas por los docentes-investigadores de los Institutos de Investigación de la Facultad de Medicina, y existiendo la inquietud por conocer con mayor profundidad la actividad e impacto de la investigación y publicación en la Facultad de Medicina, el objetivo del presente trabajo fue determinar la actividad de investigación y publicación de los docentes-investigadores de los Institutos de Investigación de la Facultad de Medicina UNMSM: Instituto de Biología Andina, Instituto de Cirugía Experimental, Instituto de Ética en Salud, Instituto de Investigaciones Clínicas, Instituto de Medicina Tropical e Instituto de Patología, y el Centro de Investigación en Bioquímica y Nutrición, durante los años 2004 a 2009.

\section{MÉTODOS}

El presente fue un estudio observacional, descriptivo, de corte transversal, que utilizó las Fichas para Investigadores elaborada el año 2009 por la Unidad de Investigación de la Facultad de Medicina UNMSM, con aprobación de los Directores de los Institutos de Investigación de Biología Andina (INBA), Cirugía Experimental (IICE), Ética en Salud (IES), Investigaciones Clínicas (IIC), Medicina Tropical (IMT), Patología (IP) y el Centro de Investigación en Bioquímica y Nutrición (CIBN), a raíz de las conclusiones del Taller Análisis Estratégico de Investigación, or- ganizado por la Facultad de Medicina en colaboración con la Organización Panamericana de la Salud, en abril del mismo año.

La Ficha del Investigador fue remitida a los miembros docentes investigadores de los diversos institutos de investigación, para ser llenada. Esta incluía datos personales, académicos e institucionales, relación y referencia bibliográfica de investigaciones como autor principal y como coautor durante los años 2004 a 2009, financiamientos, puntaje del Vicerrectorado de Investigación, publicaciones, impacto, premios, entre otros. Se definió como docente-investigador a todos los docentes inscritos en los Institutos de Investigación de la Facultad, con puntaje en el Consejo Superior de Investigación de la UNMSM y que permanecía activo hasta la elaboración de este proyecto de investigación. Se consideró la variable 'Sin dato', cuando este no había sido registrado, y 'No aplica', cuando no tocaba procesar esa información.

Se elaboró una base de datos que incluía las variables de edad en quinquenios, sexo, pertenencia a Institutos/ Centro, número de investigaciones y publicaciones realizadas los años 2004 a 2009 y la repercusión de las mismas. Para el análisis y proceso de datos se utilizó el paquete estadístico Epi.Info 3.5.1. Se utilizó estadística descriptiva y análisis de varianza para el procesamiento de datos. Se consideró significancia estadística si $\mathrm{p}<0,05$.

\section{RESULTADOS}

Luego de realizado un control de calidad de los datos, y eliminar fichas en las que los datos mostraban incoherencias, o no había información completa, el estudio incluyó 54 fichas de investigadores de cinco Institutos y el Centro de Investigación, es decir, respondieron correctamente 54 investigadores de los 116 miembros registrados (46,6\%). Remitieron la encuesta los 17 miembros del CIBN (100\%), 16 (80\%) del IMT, 
Tabla 1. Número de investigaciones $(n=659)$ realizadas por 54 investigadores de los institutos de investigación de la Facultad de Medicina, UNMSM, 2004-2009.

\begin{tabular}{ccc}
$\begin{array}{c}\text { Número de } \\
\text { Investigaciones }\end{array}$ & $\begin{array}{c}\text { Número de } \\
\text { Investigadores }\end{array}$ & $\begin{array}{c}\text { Porcentaje de } \\
\text { Investigaciones (\%) }\end{array}$ \\
0 & 2 & 3,8 \\
1 a 4 & 8 & 13,9 \\
5 a 9 & 20 & 37,1 \\
10 a 14 & 8 & 14,9 \\
15 a 19 & 5 & 9,4 \\
20 a 24 & 4 & 6,5 \\
25 a 29 & 2 & 3,8 \\
30 a 34 & 3 & 5,6 \\
35 a 56 & 2 & 3,8 \\
\hline Total & 54 & 100 \\
\hline
\end{tabular}

7 (29\%) del IES, 6 (40\%) del IBA, 6 (24\%) del IIC y 2 (18\%) del IP. No respondieron los miembros del Instituto de Cirugía Experimental.

La edad del investigador varió entre 30 y 78 años, siendo la media 56,9 años. El sexo de quienes respondieron fue masculino en $51,9 \%{ }^{(28)}$ y femenino en $48,1 \%{ }^{(26)}$. En la categoría docente, $27(50 \%)$ eran profesores principales, $23(42,6 \%)$ asociados y $4(7,4 \%)$ auxiliares; $13(24,1 \%)$ estaban a dedicación exclusiva, $27(50 \%)$ a tiempo completo y $14(25,9 \%)$ a tiempo parcial. De 32 profesores con grado académico, 18 (56,3\%) habían alcanzado el grado de doctor y $14(43,8 \%)$ de magíster. La mayoría era médico ${ }^{(20)}$, de ellos eran médicos especialistas $15 \quad(27,8 \%)$ y médicos generales 5 (9,3\%); biólogos (14; 25,9\%), químico farmacéuticos 8 (14,8\%), tecnólogos médicos 7 (13\%), 3 enfermeras y 2 nutricionistas.

El número de investigaciones fue 659 trabajos durante los seis años (tabla 1$)$, varió entre ninguna $(2 ; 3,8 \%)$ y $56(1 ; 1,9 \%)$, siendo la media 12,2 investigaciones en el lapso estudiado (2004-2009) por cada investigador. Y el promedio por cada investigador durante los 6 años de estudio fue de 2 investigaciones por año. La mitad de docentes-investigadores realizaron entre 0 y 6 investigaciones durante los 6 años.
La autoría principal de los trabajos varió entre ninguna $(7 ; 13 \%)$ y hasta en 11 investigaciones $(2 ; 3,7 \%)$, con un promedio de 4 autorías principales en el lapso estudiado. El promedio de coautoría fue 6,8. Los investigadores tenían un promedio de investigaciones con incentivo al investigador y con incentivo a la investigación de 11,5 (40 investigaciones Con Con), sin incentivo al investigador y con incentivo a la investigación 0,3 (3 investigaciones Sin Con) y sin incentivo al investigador y sin incentivo a la investigación 3,8 (17 investigaciones Sin Sin). En total, recibieron financiamiento para sus investigaciones 44 (81,5\%). Tuvieron que disminuir su carga docente de manera de poder investigar $14(25,9 \%)$.

En la tabla 2 observamos el número de investigaciones publicadas y su relación con el número de investigadores que la realizaron. Con 393 publicaciones durante los años 2004-2009, el promedio para dicho periodo fue 7,2 publicaciones, siendo la media $1,2 \mathrm{pu}-$ blicaciones por investigador/año.

De las 393 publicaciones, 343 $(87,3 \%)$ aparecieron en revistas nacionales y $50(12,7 \%)$ en revistas internacionales. Publicaron en revista nacional un promedio de 6,6 investigadores y en revista internacional 1,6 investigadores durante los años 2004-2009; 41 de quienes respondieron $(75,9 \%)$ aseguraron que alguna de sus publicaciones había tenido impacto, principalmente en salud (35; 89,7\%), en educación (3; $7,7 \%)$ o en beneficio de la sociedad (1; $2,6 \%)$. Diez investigadores (18,5\%) no realizaron publicación alguna en el lapso estudiado. El puntaje asignado a las publicaciones científicas registradas en el Consejo Superior de Investigaciones era en promedio 37,9 , variando entre 2 y 99,9 .

No se halló diferencia significativa entre el número de investigaciones realizadas y el género del investigador, el grado académico, la clase docente, la categoría docente, la profesión o Instituto de Investigación al que pertenecían, aunque el promedio de investigaciones del Instituto de Investigaciones Clínicas fue mayor al resto.

Tabla 2. Investigaciones publicadas $(n=393)$ por 54 investigadores de los Institutos de investigación de la Facultad de Medicina UNMSM 2004-2009.

\begin{tabular}{ccc}
$\begin{array}{c}N^{0} \text { investigaciones } \\
\text { publicadas }\end{array}$ & Investigadores & $\begin{array}{c}\text { Porcentaje } \\
\%\end{array}$ \\
0 & 10 & 18,5 \\
1 & 3 & 5,6 \\
2 & 3 & 5,6 \\
3 & 3 & 5,6 \\
4 & 3 & 5,6 \\
5 & 7 & 13,0 \\
6 & 9 & 16,7 \\
7 & 3 & 5,6 \\
8 & 2 & 3,7 \\
9 & 1 & 1,9 \\
10 & 2 & 3,7 \\
11 & 1 & 1,9 \\
12 & 1 & 1,9 \\
14 & 1 & 1,9 \\
19 & 2 & 3,7 \\
26 & 1 & 1,9 \\
51 & 1 & 1,9 \\
56 & 1 & 1,9 \\
\hline 393 & 54 & 100 \\
\hline
\end{tabular}




\section{DISCUSIÓN}

En el estudio para conocer la producción de cada docente-investigador, entre los años 2004 y 2009, a través de una Ficha de Registro del Investigador - 2009 diseñada para la tarea, contestó la mitad de los miembros permanentes de 5 Institutos y el CIBN, en proporciones diferentes, no habiéndose recibido respuestas del Instituto de Investigación de Cirugía Esperimental.

La edad del docente-investigador tuvo una media de 56,9 años, edad similar a la encontrada en los docentes de la Facultad en un artículo sobre producción científica de los investigadores de nuestra Facultad ${ }^{(6)}$. Estudios en Canadá encuentran que conforme la edad de los investigadores pasa los 50 años, sus artículos son cada vez más reconocidos en revistas de calidad y son también más citados, a pesar de su disminución en productividad. Por lo tanto, los investigadores de toda edad tienen un rol significativo en la dinámica científica ${ }^{(7)}$.

No hubo diferencia de sexo, la mitad era profesor principal y $43 \%$ asociado, estando la cuarta parte nombrado a dedicación exclusiva, $50 \%$ a tiempo completo y $26 \%$ a tiempo parcial. De los 32 (59\%) profesores que tenían grado académico, $56 \%$ había alcanzado el grado de doctor y $44 \%$ el de magíster, indicando que la mayoría de docentesinvestigadores se había preocupado de alcanzar el grado académico de doctor o magíster, o que posiblemente lo alcanzó por medio de la investigación, entre otros méritos. La mayoría de ellos era médico o biólogo.

El número de investigaciones entre 2004 al 2009 fue 659 y varió entre ninguna $(2 ; 3,7 \%)$ y $56(1 ; 1,9 \%)$ por investigador, siendo el promedio 2 investigaciones por investigador/año. La mitad de docentes-investigadores había realizado entre 0 y 6 investigaciones durante los 6 años. El 87\% había sido autor principal, acumulándose en dos investigadores $(3,7 \%)$ la autoría principal de 11 investigaciones cada uno. El promedio resultó en 4 autorías principales en el lapso, es decir, 0,7 autorías principales de investigación por año. Cuarentiún investigadores fueron coautores en 327 trabajos, con media de 7,9 y promedio de 1,3 coautorías por investigador/año.

Una de las inquietudes presentadas en el Taller de 2009 motivo de la presente investigación, fue que los docentes no tenían tiempo suficiente para investigar. El estudio encontró que 14 $(25,9 \%)$ docentes tuvieron que disminuir su carga docente de manera de poder investigar. Somos testigos de que muchos docentes investigadores tienen carga académica intensa en diversas escuelas académico profesionales y departamentos académicos.

Toda investigación realizada y que no se publica, queda prácticamente en el olvido ${ }^{(8-10)}$. Es indispensable la diseminación de los resultados de las investigaciones, en particular en el área de salud ${ }^{(11,12)}$.En el lapso de estudio, se halló que los investigadores publicaron 393 artículos, variando entre ninguno $(10 ; 18,5 \%)$ y $56(1 ; 1,9 \%)$ artículos, con una media de 7,3. La autoría principal de los trabajos varió entre ninguna $(7 ; 13 \%)$ y 11 investigaciones (2; 3,7\%), con un promedio de 4 autorías principales en el lapso estudiado, mientras que el promedio de coautoría fue 6,8 . Publicaron $87,3 \%$ en revistas nacionales y $12,7 \%$ en revistas internacionales en los seis años de estudio, es decir, siete veces más en revistas nacionales.

Podemos señalar que las investigaciones en salud como en otras requieren el compromiso, cooperación y dedicación de varias personas, y muchas veces son estudios multicéntricos ${ }^{(13)}$, como ocurrió con varios trabajos. Sin embargo, también existe la autoría múltiple injustificada en las investigaciones y artículos científicos ${ }^{(14,15)}$, que ha sido preocupación también de nuestras autoridades universitarias.

A la variable sobre el impacto de alguna de las publicaciones realizadas, $76 \%$ aseguró que sí habían tenido impacto, principalmente en salud (90\%) y menos en educación y salud. Esta afirmación requeriría un estudio particular para determinar el concepto y la magnitud del impacto.

En síntesis, los 54 docentes-investigadores que remitieron su ficha del investigador llenada señalaron que habían realizado 659 trabajos de investigación durante los seis años de estudio, con un promedio de 2 investigaciones por año por investigador. Tuvieron un total de 393 publicaciones, un promedio de 7,3 investigaciones en los seis años, o 1,2 por investigador-año. Diecinueve por ciento de los investigadores no realizó publicación alguna en dicho lapso.

No se halló diferencia significativa de género del investigador, grado académico, clase docente, profesión o Instituto/Centro, aunque el promedio de investigaciones del Instituto de Investigaciones Clínicas pareció mayor al resto, pero solo había remitido la ficha la cuarta parte de los miembros.

Queda por conocer la actividad de investigación y publicación de la mitad de docentes investigadores que no remitieron su ficha, a pesar de múltiples requerimientos, a través de los Directores de los Institutos/Centro. Los resultados podrían tener alguna modificación, aunque el avance de un estudio paralelo realizado en el Taller de Investigación 2010, del Vicerrectorado de Investigación y Consejo Superior de Investigaciones, los resultados referidos a investigadores fueron similares a los encontrados en el presente trabajo (estudio aún no publicado).

En conclusión, la investigación realizada sobre las actividades del investigador de la Facultad de Medicina por medio de una Ficha del investigador 2009, tuvo respuesta de la mitad de los miembros de tanto el Centro de Investigación de Bioquímica y Nutrición como de cinco de los seis Institutos de Investigación. La edad promedio de los investigadores fue 57 años, que entre 2004 y 2009 realizaron 659 trabajos de investigación, siendo la media de 2 investigaciones por investigador/año. Los 393 artículos publicados representaron 
1,2 publicaciones por investigador-año; 19\% de los investigadores no realizó publicación alguna en dicho lapso.

Sugerimos continuar este tipo de estudios sobre investigación y publicación en la Facultad de Medicina, que pueda evaluar dichas actividades y compararlas con los resultados de otras facultades de la UNMSM, así como de otras universidades nacionales y particulares. Con seguridad, ello fomentará un mayor número y calidad de nuestra investigación y publicación, esencial para la acreditación de la Facultad de Medicina. Además, observamos cada vez mayor interés en la investigación en los planes del gobierno ${ }^{(16,17)}$, financiamiento ${ }^{(18)}$, así como en las discusiones internacionales sobre desarrollo ${ }^{(19)}$. Recordemos que nuestros países tienen responsabilidad en la formación profesional en salud ${ }^{(20)}$-que incluye la investigación y publicación- en una época de globalización y migración ${ }^{(21)}$ y de enfermedades emergentes y reemergentes ${ }^{(22)}$.

\section{REFERENCIAS BIBLIOGRÁFICAS}

1. Ministerio de Agricultura. Instituto Nacional de Innovación Agraria - INIA. Reglamento de Organización y Funciones. Glosario de términos. Disponible en: http://www.inia.gob.pe/institucional/ ROF/Terminos.pdf

2. Real Academia Española. Diccionario de la Lengua Española, vigésima segunda edición. Disponible en: http://buscon.rae.es/drael/
3. Hernández R, Fernández C, Baptista M. Metodología de la Investigación. Quinta edición. México: McGraw Hill. 2010:613 pags.

4. Panamerican Health Organization. Aplicación del método epidemiológico a la investigación en salud ocupacional. Disponible en: http://bvs.per.paho.org/ cursoepi/e/modulo2.html.

5. Vicerrectorado de investigación, UNMSM. Reseña. Dsiponible en: http://www.vicerrectoradounmsm. bravehost.com/.

6. Sogi C, Perales A, Anderson A, Bravo E. El proceso de producción científica de los investigadores de la Facultad de Medicina, UNMSM. An Fac med. 2002;63(2):31-40.

7. Gingras $Y$, Lariviere V, Macaluso B, Robitaille J-P. The effects of aging on researchers' publication and citation patterns. Plos one. 2008;3(12):e4048.

8. Day RA. Cómo escribir y publicar trabajos científicos (526). Phoenix: The Orix Press (1979). 3era. edición en español, 1990. $214 \mathrm{p}$

9. Home PD. Técnicas para asegurarse de que su próximo trabajo nunca se llegue a publicar. En: OPS. Publicación científica: aspectos metodológicos, éticos y prácticos en ciencias de la salud (550) Washington: OPS, 1994: 105-9.

10. Pamo OG. Editorial. Publicar o perecer. Bol Soc Peru Med Int. 2000;13:180-1.

11. Abrahams N, Adhikari R, Bhagwat IP, Christofides $\mathrm{N}$, Djibuti M. Changing the debate about health research for development. J Public Health Policy. 2004;25:259-87.

12. Huamani C. Pacheco-Romero J. Visibilidad y producción de las revistas biomédicas peruanas. Rev Gastroenterol Perú. 2009;29(2):132-9.

13. Flanagin A, Fontanarosa PB, DeAngelis CD. Authorship for research groups. JAMA. 2002;288(24):3166-8.

14. Silva GA. La autoria múltiple y la autoria injustificada en los artículos científicos. Investigación en Salud. 2005;7(2):84-90.

15. Davidoff F, for the CSE Task Force on Authorship. Who's the author? Problems with biomedical authorship, and some possible solutions. Science Editor. 2000;23:111-9.

16. Ministerio de Salud. Prioridades de investigación en salud en el Perú: análisis del proceso. Lima, Perú: Instituto Nacional de Salud; 2007. Disponible en: http://www.ins.gob.pe/insvirtual/images/otrpubs/pdf/Prioridades $\% 20$ de $\% 20$ Investigaci\%C3\%B3n\%20en\%20Salud.pdf.
17. Cabezas C. Rol del Estado en la investigación científica en salud y transparencia en la información, Rev Peru Med Exp Salud Publica. 2006;23(4): 275-83.

18. Cáceres CF, Mendoza W. Globalized research and "national science": the case of Peru. Am J Publ Health. 2009;99(10):1792-8.

19. Cáceres CF, Cuba V, eds. Investigación Científica y Políticas Públicas en Salud: Memoria de las I Jornadas Científicas en Salud Pública. Lima, Perú: Universidad Peruana Cayetano Heredia, Facultad de Salud Pública, 2007. Disponible en: http://bvs. minsa.gob.pe/local/MINSA/1054_SP113.pdf.

20. 20. Murillo JP, Mendoza W, Franco G. Quo vadis salud publica? An Fac med. 2000;61(2):146-60.

21. Sotomayor R. Globalización y la responsabilidad de los países en desarrollo: el caso de la migración de los profesionales de salud. Rev Peru Med Exp Salud Publica. 2007;24(3):300-6.

22. Cueto M, Zamora V, eds. Historia, Salud y Globalización. Lima, Perú: Universidad Peruana Cayetano Heredia, Instituto de Estudios Peruanos; 2006.

Articulo presentado el 16 de octubre de 2011 y aceptado para publicación el 11 de enero de 2012.

Financiamiento: Investigación autofinanciada. Proyecto de investigación Sin Sin del Vicerrectorado de Investigación, aprobado por RD 0409-D-FM2010.

Conflictos de intereses: El Dr. José Pacheco ha sido Director de la Unidad de Investigación y es Miembro del Instituto de Investigaciones Clínicas de la Facultad de Medicina, UNMSM. La Mg. Doris Huerta fue Directora del Centro de Investigación en Bioquímica y Nutrición, Facultad de Medicina, UNMSM. El Dr. Carlos Galarza fue Director del Instituto de Investigaciones Clínicas, Facultad de Medicina, UNMSM.

Correspondencia:

Dr. José Pacheco Romero

Venecia 225, San Borja, Lima, Perú

Correo electrónico: jpachecoperu@yahoo.com 